\title{
The Variance of the Error Function in the Shifted Circle Problem Is a Wild Function of the Shift
}

\section{Pavel M. Bleher, Freeman J. Dyson}

School of Natural Sciences, Institute for Advanced Study, Princeton, NJ 08540, USA

Received: 8 March 1993

Abstract: We prove that the variance of the error function in the shifted circle problem, as a function of the shift, is a continuous function which has a sharp local maximum with infinite derivatives at every rational point on a plane.

\section{Introduction}

Let

$$
N(R ; \alpha)=\#\left\{m \in \mathbf{Z}^{2}:|m-\alpha| \leqq R\right\}, \quad \alpha=\left(\alpha_{1}, \alpha_{2}\right) \in \mathbf{R}^{2},
$$

be the number of lattice points inside the circle of radius $R$ with the center at $\alpha$, and

$$
F(R ; \alpha)=\frac{N(R ; \alpha)-\pi R^{2}}{R^{1 / 2}}
$$

As was shown in $[\mathrm{B}]$ and $[\mathrm{BCDL}]$, the limit,

$$
D(\alpha)=\lim _{T \rightarrow \infty} \frac{1}{T} \int_{1}^{T}|F(R ; \alpha)|^{2} d R
$$

exists and is equal to

where

$$
D(\alpha)=\left(2 \pi^{2}\right)^{-1} \sum_{n=1}^{\infty} n^{-3 / 2}\left|r_{\alpha}(n)\right|^{2}
$$

$$
r_{x}(n)=\sum_{k^{2}+l^{2}=n} e\left(k \alpha_{1}+l \alpha_{2}\right), \quad e(t)=\exp (2 \pi i t) .
$$

For $\alpha=0$ (1.1) reduces to a classical result of Cramér (see [C]). After averaging (1.1) in $\alpha$ we get a formula of Kendall (see $[\mathrm{K}]$ ):

$$
\int_{0}^{1} \int_{0}^{1} D(\alpha) d \alpha=\left(2 \pi^{2}\right)^{-1} \sum_{n=1}^{\infty} n^{-3 / 2} r_{0}(n) .
$$


$D(\alpha)$ is the mean value of $F^{2}(R ; \alpha)$, and it is also equal to

$$
D(\alpha)=\int_{-\infty}^{\infty} x^{2} v_{\alpha}(d x)
$$

where $v_{\alpha}(d x)$ is the limit distribution of $F(R ; \alpha)$,

$$
\lim _{T \rightarrow \infty} \frac{1}{T} \int_{\{R: a \leqq F(R ; \alpha) \leqq b ; 1 \leqq R \leqq T\}} d R=\int_{a}^{b} v_{\alpha}(d x)
$$

(see $[\mathrm{B}, \mathrm{BCDL}])$. In addition,

$$
\lim _{T \rightarrow \infty} \frac{1}{T} \int_{1}^{T} F(R ; x) d R=\int_{-\infty}^{\infty} x v_{x}(d x)=0
$$

hence $D(\alpha)$ is the variance of the limit distribution of $F(R ; \alpha)$. The existence of a limit distribution of $F(R ; \alpha)$ for $\alpha=0$ was proved by Heath-Brown (see [H-B]).

Since the series in the RHS of (1.1) is uniformly convergent, $D(\alpha)$ is continuous in x. Here we prove

Theorem 1.1 For every rational $\beta \in \mathbf{Q}^{2}$,

$$
\lim _{\gamma \rightarrow \beta}(|\log | \alpha-\beta|| \cdot|\alpha-\beta|)^{-1}(D(\alpha)-D(\beta))=-C(\beta), \quad C(\beta)>0 .
$$

Since $D(\alpha)$ has a sharp local maximum with infinite derivatives at every rational point, it is a wild function. By wild function we mean a continuous function which is nondifferentiable on a dense set. $C(\beta)$ is defined as follows. Let $Q$ be the integer such that

$$
2 Q \beta_{1}=n_{1}, \quad 2 Q \beta_{2}=n_{2}
$$

are integers and there is no common factor dividing all three of $Q, n_{1}, n_{2}$. Then

where

$$
C(\beta)=C\left(16 / \pi^{2}\right)(Q r(Q))^{-1}
$$

$$
r(Q)=\prod_{p \mid Q}\left(1+p^{-1}\right)
$$

with the product taken over primes $p$ dividing $Q$, and

$$
\begin{aligned}
& C=3(Q \text { even }), \quad C=4\left(Q \text { odd },\left(n_{1}+n_{2}\right) \text { even }\right), \\
& C=2\left(Q \text { odd },\left(n_{1}+n_{2}\right) \text { odd }\right) .
\end{aligned}
$$

$D(\alpha)$ achieves its global maximum at $\alpha=m$ and $\alpha=m+(1 / 2,1 / 2), m \in \mathbf{Z}^{2}$. Indeed,

$$
\left|r_{\alpha}(n)\right|^{2}=\left|\sum_{k^{2}+l^{2}=n} e\left(k \alpha_{1}+l \alpha_{2}\right)\right|^{2}=\left|\sum_{k^{2}+l^{2}=n} \cos \left(2 \pi k \alpha_{1}\right) \cos \left(2 \pi l \alpha_{2}\right)\right|^{2}
$$

is maximum when

$$
\left|\cos \left(2 \pi k \alpha_{1}\right)\right|=\left|\cos \left(2 \pi l \alpha_{2}\right)\right|=1
$$

and $\operatorname{sign}\left(\cos \left(2 \pi k \alpha_{1}\right) \cos \left(2 \pi l \alpha_{2}\right)\right)$ is the same for all $k, l$ with $k^{2}+l^{2}=n$. This holds for all $n \in \mathbf{N}$ iff $\alpha=m$ or $\alpha=m+(1 / 2,1 / 2), m \in \mathbf{Z}^{2}$. 
It is to be noted that the wild behavior of $D(\alpha)$ is closely related to a bumpy shape of the exponential sum

$$
S_{\alpha}(b)=\sum_{n=1}^{\infty}\left|r_{\alpha}(n)\right|^{2} \exp (-n / b),
$$

as a function of $\alpha$, when $b \rightarrow \infty$ (see [BD]). $S_{\alpha}(b)$ is a key tool used to study the limit distribution of $F(R ; \alpha)$ in [BCDL].

As a generalization of Theorem 1.1 consider the variance

$$
D_{I}(\alpha)=\lim _{T \rightarrow \infty} \frac{1}{T} \int_{1}^{T}\left|F_{I}(R ; \alpha)\right|^{2} d R,
$$

for a general lattice-point problem, with

where

$$
F_{I}(R: \alpha)=\frac{N_{I}(R ; \alpha)-A R^{2}}{R^{1 / 2}}
$$

$$
N_{I}(R ; \alpha)=\#\left\{m \in \mathbf{Z}^{2}: I(m-\alpha) \leqq R^{2}\right\}, \quad \alpha \in \mathbf{R}^{2} ; \quad A=\text { Area }\{x: I(x) \leqq 1\},
$$

and $I(x)>0$ is an arbitrary $C^{\infty}$ positive convex homogeneous of order 2 function on $\mathbf{R}^{2} \backslash\{0\}$. As was proved in [B] the limit (1.7) always exists and is equal to

where

$$
D_{I}(\alpha)=\left(2 \pi^{2}\right)^{-1} \sum_{n=1}^{\infty}\left|u_{\gamma}(n)\right|^{2}
$$

$$
u_{\alpha}(n)=\sum_{k, l: J(k, l)=J_{n}}\left|k^{2}+l^{2}\right|^{-3 / 4} \sqrt{\rho(k, l)} e\left(k \alpha_{1}+l \alpha_{2}\right),
$$

$0=J_{0}<J_{1}<J_{2}<\cdots$ are all possible values of

$$
J(k, l)=\max _{x_{1}, x_{2} \in \mathbf{R}}\left[2\left(k x_{1}+l x_{2}\right)-I\left(x_{1}, x_{2}\right)\right], \quad k, l \in \mathbf{Z},
$$

and $\rho(k, l)$ is the curvature of the curve $\Gamma=\{x: I(x)=1\}$ at the point $x \in \Gamma$ where $\operatorname{grad} I(x)$ is collinear to the vector $(k, l)$. The formula (1.8) is a generalization of (1.1).

By $(1.8) D_{I}(\alpha)$ is independent of $\alpha$ if for every $n=1,2, \ldots$, the set of $(k, l)$ such that $J(k, l)=J_{n}$ consists of one point. This can be viewed as a "generic" case of $I(x)$, so that "generically" $D_{I}(\alpha)$ is constant in $\alpha$. On the other hand, if $I(x)$ possesses some symmetry, say, $I(-x)=I(x)$, then $D_{I}(\alpha)$ is, in general, nonsmooth, since the Fourier coefficients of $D_{I}(\alpha)$ in (1.8) decay slowly. For instance, if $I(x)=\left(x_{1} /\right.$ $a)^{2}+\left(x_{2} / b\right)^{2}$ and $a^{2} / b^{2}$ is irrational then $J(k, l)=(a k)^{2}+(b l)^{2}$ and $J\left(k_{1}, l_{1}\right)=J\left(k_{2}, l_{2}\right)$ iff $k_{1}= \pm k_{2}, l_{1}= \pm l_{2}$. In this case it is not difficult to prove that $D_{I}(\alpha)$ is nondifferentiable at half-integer points $\alpha \in(1 / 2) Z^{2}$. More precisely,

$$
D_{I}(\alpha)=-C \sqrt{\frac{\sin ^{2} 2 \pi \alpha_{1}}{a^{2}}+\frac{\sin ^{2} 2 \pi \alpha_{2}}{b^{2}}}+R_{I}(\alpha),
$$

where $C>0$ and $R_{I}(\alpha)$ is differentiable everywhere.

In addition, an "arithmetic" degeneracy of $J(k, l)$ can worsen the smoothness of $D_{I}(\alpha)$. For instance, if $I(x)=\left(x_{1} / a\right)^{2}+\left(x_{2} / b\right)^{2}$ and $a^{2} / b^{2}$ is rational, then $\#\left\{(k, l): J(k, l)=J_{n}\right\}$ is unbounded as $n \rightarrow \infty$. This "arithmetic" degeneracy of $J(k, l)$ causes wild nonsmoothness of the variance $D_{I}(\alpha)$, namely, with the help of the 
same method that we use in the proof of Theorem 1.1, we can prove that for every rational $\beta \in \mathbf{Q}^{2}$,

$$
\lim _{\alpha \rightarrow \beta}(|\log | \alpha-\beta|| \cdot|\alpha-\beta|)^{-1}\left(D_{I}(\alpha)-D_{I}(\beta)\right)=-C_{I}(\beta), \quad C_{I}(\beta)>0 .
$$

The set-up of the remainder of the paper is the following. In Sect. 2 we prove some preliminary results for Theorem 1.1. The proof of Theorem 1.1 is slightly different for $\beta=0$ and for $\beta \neq 0$. In Sect. 3 we prove Theorem 1.1 for $\beta=0$, and in Sect. 4 we prove Theorem 1.1 for $\beta \neq 0$.

Throughout the paper $C, C_{0}, C_{1}, \ldots$ are considered to be fixed positive constants. However they often change value from one equation to the next.

\section{Preliminaries}

This section consists of identities valid for all $\alpha$. The sum (1.1) may be written

$$
\begin{aligned}
D(\alpha) & =\left(2 \pi^{2}\right)^{-1} \sum_{n=1}^{\infty} n^{-3 / 2}\left|\sum_{m \in \mathbf{Z}^{2}:|m|^{2}=n} e(\alpha \cdot m)\right|^{2} \\
& =\left(2 \pi^{2}\right)^{-1} \sum_{m m^{\prime}}|m|^{-3} e\left(\alpha \cdot\left(m-m^{\prime}\right)\right)
\end{aligned}
$$

summed over integer vectors $m, m^{\prime}$ with $m^{2}=m^{\prime 2}$. The sum (2.1) may be converted into an unrestricted sum (see Appendix B in [BCDL]),

$$
D(\alpha)=2 \pi^{-2} \sum_{j h k l} e\left(h\left(l \alpha_{1}-k \alpha_{2}\right)\right)\left(\left(j^{2}+h^{2}\right)\left(k^{2}+l^{2}\right)\right)^{-3 / 2},
$$

summed over all $(j, h, k, l) \in \mathbf{Z}^{4}$ satisfying

$$
\begin{gathered}
j^{2}+h^{2} \neq 0, \quad k^{2}+l^{2} \neq 0, \\
\text { either } j \equiv h \equiv 0, \text { or } j \equiv h \equiv k \equiv l \equiv 1(\bmod 2),
\end{gathered}
$$

and

$$
k, l \text { are relatively prime }
$$

which means that either $|k|+|l|=1$, or $\operatorname{gcd}(|k|,|l|)=1$.

According to two possibilities allowed by $(2.4)$, we divide $D(\alpha)$ into two parts,

$$
D(\alpha)=D_{\mathrm{e}}(\alpha)+D_{\mathrm{o}}(\alpha)
$$

where the terms with $j$ and $h$ even are

$$
D_{\mathrm{e}}(\alpha)=\sum_{k l} U(w)\left(k^{2}+l^{2}\right)^{-3 / 2}
$$

summed over integers $(k, l)$ satisfying (2.5), and the terms with $j$ and $h$ odd are

$$
D_{\mathrm{o}}(\alpha)=\sum_{\text {odd } k l} V(w)\left(k^{2}+l^{2}\right)^{-3 / 2}
$$

summed over odd integers $k$ and $l$ satisfying (2.5). The functions $(U, V)$ are defined by

$$
\begin{aligned}
U(w) & =(2 \pi)^{-2} \sum_{j^{2}+h^{2} \neq 0} e(h w)\left(j^{2}+h^{2}\right)^{-3 / 2} \\
& =(2 \pi)^{-2} \sum_{j^{2}+h^{2} \neq 0} \cos (2 \pi h w)\left(j^{2}+h^{2}\right)^{-3 / 2},
\end{aligned}
$$




$$
\begin{aligned}
V(w) & =(2 \pi)^{-2} \sum_{\text {half-odd-integer } j h} e(h w)\left(j^{2}+h^{2}\right)^{-3 / 2} \\
& =(2 \pi)^{-2} \sum_{\text {half-odd-integer } j h} \cos (2 \pi h w)\left(j^{2}+h^{2}\right)^{-3 / 2} .
\end{aligned}
$$

In (2.7)-(2.9) we have used the abbreviation

$$
w=2\left(l \alpha_{1}-k \alpha_{2}\right)
$$

According to (2.9),

$$
\begin{gathered}
U(w+1)=U(w), \quad V(w+1)=-V(w), \\
U(-w)=U(w), \quad V(-w)=V(w) .
\end{gathered}
$$

Lemma 2.1. $U(w)$ and $V(w)$ are infinitely differentiable on $[0,1]$, and

$$
U^{\prime}(+0)=V^{\prime}(+0)=-1 \text {. }
$$

Remark. (2.11), (2.12) imply that

$$
U^{\prime}(+0)-U^{\prime}(-0)=V^{\prime}(+0)-V^{\prime}(-0)=-2 .
$$

We shall use Lemma 2.1 with (2.11) to note that $U(w)$ and $V(w)$ are bounded absolutely; and also that $U(t)=-|t|+O\left(|t|^{2}\right)$ for sufficiently small $t$.

Proof of Lemma 2.1 is given in Appendix to the paper. We are now ready to prove Theorem 1.1. The proof is slightly different for $\beta=0$ and $\beta \neq 0$. First we consider $\beta=0$.

\section{Proof of Theorem 1.1 for $\beta=0$}

Let $\zeta_{i}=|\alpha|^{-1} \alpha_{i}, i=1,2,|\alpha|=\left(\alpha_{1}^{2}+\alpha_{2}^{2}\right)^{1 / 2}$, and $\zeta=\left(\zeta_{1}, \zeta_{2}\right)$, so that $\alpha=|\alpha| \zeta$, $|\zeta|=1$. Then from (2.7),

$$
\begin{aligned}
|\alpha|^{-1}\left(D_{\mathrm{e}}(\alpha)-D_{\mathrm{e}}(0)\right) & =|\alpha|^{-1} \sum_{k l}\left(U\left(2\left(l \alpha_{1}-k \alpha_{2}\right)\right)-U(0)\right)\left(k^{2}+l^{2}\right)^{-3 / 2} \\
& =\sum_{k l}\left(U\left(2\left(l|\alpha| \zeta_{1}-k|\alpha| \zeta_{2}\right)\right)-U(0)\right)\left((k|\alpha|)^{2}+(l|\alpha|)^{2}\right)^{-3 / 2}|\alpha|^{2} \\
& =\sum_{k l} \Phi(|\alpha| k,|\alpha| l)|\alpha|^{2} \equiv I(|\alpha|)
\end{aligned}
$$

which is an approximating sum for the integral

with

$$
I=\left(6 / \pi^{2}\right) \int_{-\infty}^{\infty} \int_{-\infty}^{\infty} \Phi\left(x_{1}, x_{2}\right) d x_{1} d x_{2}
$$

$$
\Phi\left(x_{1}, x_{2}\right)=\left(U\left(2\left(x_{2} \zeta_{1}-x_{1} \zeta_{2}\right)\right)-U(0)\right)\left(x_{1}^{2}+x_{2}^{2}\right)^{-3 / 2} .
$$

The summation in (3.1) goes over relatively prime $k, l$, and the factor $\left(6 / \pi^{2}\right)$ in (3.2) is the density of pairs $(k, l)$ with relatively prime $k, l$.

By Lemma 2.1, for small $|t|, U(t)-U(0) \sim-|t|$. This implies that the integral (3.2) diverges logarithmically at the origin. The approximating sum (3.1) is taken over points $(|\alpha| k,|\alpha| l) \neq 0$ which belong to the lattice with the space $|\alpha|$, so we may expect that $I(|\alpha|)$ behaves like $C|\log | \alpha||$. 
We can estimate easily the part of the sum in (3.1) with $k^{2}+l^{2} \geqq|\alpha|^{-2}$. Lemma 2.1 implies that $U(t)$ is bounded, so this part is

$$
\begin{aligned}
\sum_{k^{2}+l^{2} \geqq|\alpha|^{-2}} \Phi(|\alpha| k,|\alpha| l)|\alpha|^{2} \mid & \leqq \sum_{k^{2}+l^{2} \geqq|\alpha|^{-2}}|\Phi(|\alpha| k,|\alpha| l)||\alpha|^{2} \\
& \leqq C \sum_{k^{2}+l^{2} \geqq|\alpha|^{-2}}\left((|\alpha| k)^{2}+(|\alpha| l)^{2}\right)^{-3 / 2}|\alpha|^{2} \\
& =C|\alpha|^{-1} \sum_{k^{2}+l^{2} \geqq|\alpha|^{-2}}\left(k^{2}+l^{2}\right)^{-3 / 2} \leqq C_{0} .
\end{aligned}
$$

Let us fix large numbers $M, N$, which will be chosen later, and consider a sequence of squares

$$
S_{i}=\left\{\left|x_{1}\right|,\left|x_{2}\right| \leqq M_{i}=M((N+1) / N)^{i}\right\}, \quad i \geqq 0 .
$$

We shall consider $S_{i}$ with $i=0,1, \ldots, p$, where $p$ is chosen in such a way, that

$$
M_{p-1} \leqq|\alpha|^{-1}<M_{p} \text {, }
$$

or in other words,

$$
p=\left[\left(\log \left(1+N^{-1}\right)\right)^{-1}|| \log |\alpha||-\log M|\right]+1 .
$$

The choice of $S_{i}$ ensures the following property of commensurability of $S_{i}$ and $S_{i+1}$ : We can partition $S_{i}$ into $4 N^{2}$ squares of side $N^{-1} M_{i}$, and $S_{i+1}$ into $4(N+1)^{2}$ squares of the same side. This implies that the square annulus $S_{i+1} \backslash S_{i}$ is partitioned into $4(2 N+1)$ squares $S_{i j}$ of side $N^{-1} M_{i}$. Let $m_{i j}$ be the center of $S_{i j}$.

Consider the sum

$$
I_{i j}(|\alpha|)=\sum_{S_{i j}} \Phi(|\alpha| m)|\alpha|^{2}
$$

where the summation goes over relatively prime $k, l$ with $m=(k, l) \in S_{i j} . I_{i j}(|\alpha|)$ is the $S_{i j}$-part of the sum $I(|\alpha|)$ in (3.1). We want to compare $I_{i j}(|\alpha|)$ first with

$$
J_{i j}(|\alpha|)=\Phi\left(|\alpha| m_{i j}\right)|\alpha|^{2} \sum_{S_{i j}} 1
$$

and then with

where

$$
I_{i j}=\left(6 / \pi^{2}\right) \int_{X_{i j}} \Phi(x) d x
$$

$$
X_{i j}=|\alpha| S_{i j}=\left\{x=|\alpha| y, y \in S_{i j}\right\} .
$$

Denote by $x_{i j}=|\alpha| m_{i j}$ the center of the square $X_{i j}$. The side of $X_{i j}$ is equal to $|\alpha| N^{-1} M_{i}$, hence for every $x \in X_{i j}$,

Let

$$
\left|x-x_{i j}\right| \leqq|\alpha| N^{-1} M_{i}
$$

so that

$$
U_{0}(x)=U\left(2\left(x_{2} \zeta_{1}-x_{1} \zeta_{2}\right)\right)-U(0)
$$

$$
\Phi(x)=U_{0}(x)|x|^{-3}
$$

By Lemma 2.1, $U(w)$ is a periodic Lipshitz function, hence

$$
\begin{aligned}
\left|U_{0}(x)-U_{0}\left(x_{i j}\right)\right| \leqq C\left|x-x_{i j}\right| \leqq C|\alpha| N^{-1} M_{i} \\
\left|U_{0}(x)\right| \leqq C|x|
\end{aligned}
$$


Also,

$$
\begin{aligned}
\left.|| x\right|^{-3}-\left|x_{i j}\right|^{-3} \mid & \leqq C\left|x-x_{i j}\right|\left|x_{i j}\right|^{-4} \leqq C_{0}|\alpha| N^{-1} M_{i}\left(|\alpha| M_{i}\right)^{-4} \\
& =C_{0}|\alpha|^{-3} N^{-1}\left(M_{i}\right)^{-3}
\end{aligned}
$$

When $x \in X_{i j}$,

Therefore,

$$
\begin{aligned}
\left|\Phi(x)-\Phi\left(x_{i j}\right)\right| & \leqq\left|U_{0}(x)-U_{0}\left(x_{i j}\right)\right||x|^{-3}+\left.\left|U_{0}\left(x_{i j}\right)\right||| x\right|^{-3}-\left|x_{i j}\right|^{-3} \mid \\
& \leqq C\left(|\alpha| N^{-1} M_{i}\left(|\alpha| M_{i}\right)^{-3}+|\alpha| M_{i}|\alpha|^{-3} N^{-1}\left(M_{i}\right)^{-3}\right) \\
& =2 C N^{-1}\left(|\alpha| M_{i}\right)^{-2}
\end{aligned}
$$

$$
\begin{aligned}
\left|I_{i j}(|\alpha|)-J_{i j}(|\alpha|)\right| & =\left.\left|\sum_{S_{i j}}\left(\Phi(|\alpha| m)-\Phi\left(|\alpha| m_{i j}\right)\right)\right| \alpha\right|^{2} \mid \\
& \leqq \sum_{S_{i j}} C N^{-1}\left(|\alpha| M_{i}\right)^{-2}|\alpha|^{2} \\
& =C N^{-1}\left(M_{i}\right)^{-2} \sum_{S_{i j}} 1 \leqq C N^{-1}\left(M_{i}\right)^{-2}\left(N^{-1} M_{i}\right)^{2} \\
& =C N^{-3} .
\end{aligned}
$$

Similarly, since Area $X_{i j}=\left(|\alpha| N^{-1} M_{i}\right)^{2}$, and by (3.9), (3.11),

$$
|\Phi(x)| \leqq C|x|^{-2} \leqq C\left(|\alpha| M_{i}\right)^{-2}
$$

we obtain

where

$$
\begin{aligned}
\left|I_{i j}-J_{i j}(|\alpha|)\right|= & \mid\left(6 / \pi^{2}\right) \int_{X_{i j}}\left(\Phi(x)-\Phi\left(x_{i j}\right)\right) d x \\
& +\Phi\left(x_{i j}\right)\left(\left(6 / \pi^{2}\right)\left(|\alpha| N^{-1} M_{i}\right)^{2}-|\alpha|^{2} \sum_{\left.S_{\iota_{j}} 1\right) \mid} 1\right) \\
\leqq & C\left(N^{-1}\left(|\alpha| M_{i}\right)^{-2}\left(N^{-1}\left|M_{i}\right||\alpha|\right)^{2}\right. \\
& \left.+\left(|\alpha| M_{i}\right)^{-2}|\alpha|^{2}\left(N^{-1} M_{i}\right)^{2} \varepsilon_{M N}\right) \\
= & 2 C\left(N^{-3}+N^{-2} \varepsilon_{M N}\right),
\end{aligned}
$$

$$
\varepsilon_{M N}=\sup _{i j}\left|\left(6 / \pi^{2}\right)-\left(N^{-1} M_{i}\right)^{-2} \sum_{S_{i j}} 1\right| .
$$

Since $M_{i+1} \geqq M_{0}=M$,

$$
\lim _{M \rightarrow \infty} \varepsilon_{M N}=0
$$

To prove (3.16) let us assume that $S$ is an arbitrary square of side $a>0$ on the plane such that the origin is outside of $S$ and $\max _{x \in S}|x| \leqq L a$, where $L>0$ is a fixed number. Then by the Möbius inversion formula,

$$
\begin{aligned}
\sum_{(k, l) \in S: \operatorname{gcd}(k, l)=1} 1 & =\sum_{d=1}^{\infty} \mu(d) \sum_{(d k, d l) \in S} 1=\sum_{d=1}^{L a} \mu(d)\left[\frac{a^{2}}{d^{2}}+O\left(\frac{a}{d}\right)\right] \\
& =\sum_{d=1}^{\infty} \frac{\mu(d)}{d^{2}} a^{2}+O(a \log a)=\frac{6}{\pi^{2}} a^{2}+O(a \log a), \quad a \rightarrow \infty,
\end{aligned}
$$

where $\mu(d)$ is the Möbius function. This proves (3.16). 
From (3.12), (3.14) we obtain

$$
\left|I_{i j}(|\alpha|)-I_{i j}\right| \leqq C\left(N^{-3}+N^{-2} \varepsilon_{M N}\right) .
$$

Due to (3.7),

$$
p \leqq C N|\log | \alpha||
$$

hence (3.17) implies

$$
\begin{aligned}
\left|\sum_{i=0}^{p} \sum_{j=1}^{4(2 N+1)} I_{i j}(|\alpha|)-\sum_{i=0}^{p} \sum_{j=1}^{4(2 N+1)} I_{i j}\right| & \leqq C p\left(N^{-2}+N^{-1} \varepsilon_{M N}\right) \\
& \leqq C_{0}\left(N^{-1}+\varepsilon_{M N}\right)|\log | \alpha||
\end{aligned}
$$

or

$$
\left.\left|\sum_{S_{p} \backslash S_{0}} \Phi(|\alpha| m)\right| \alpha\right|^{2}-\left(6 / \pi^{2}\right) \int_{X_{p} \backslash X_{0}} \Phi(x) d x\left|\leqq C\left(N^{-1}+\varepsilon_{M N}\right)\right| \log |\alpha| \mid,
$$

where

$$
X_{i}=\left\{\left|x_{1}\right|,\left|x_{2}\right| \leqq|\alpha| M_{i}\right\}=|\alpha| S_{i}
$$

Notice that by (3.6),

$$
|\alpha| M_{p} \geqq 1,
$$

hence, when $m=(k, l) \in \mathbf{Z}^{2} \backslash S_{p}$, either $|k| \geqq M_{p} \geqq|\alpha|^{-1}$, or $|l| \geqq|\alpha|^{-1}$, hence by (3.4),

Similarly,

$$
\left.\left.\left|\sum_{\mathrm{Z}^{2} \backslash S_{p}} \Phi(|\alpha| m)\right| \alpha\right|^{2}\left|\leqq \sum_{|m| \geqq|\alpha|^{-1}}\right| \Phi(|\alpha|, m)|| \alpha\right|^{2} \leqq C
$$

$$
\left|\int_{\mathbf{R}^{2} \backslash X_{p}} \Phi(x) d x\right| \leqq C \int_{\{|x| \geqq 1\}}|x|^{-3} d x \leqq C_{0} .
$$

By (3.13),

$$
\left.\left.\left|\sum_{S_{0}} \Phi(|\alpha| m)\right| \alpha\right|^{2}\left|\leqq C \sum_{S_{0}}\right| m\right|^{-2} \leqq C \sum_{0<|m| \leqq 2 M}|m|^{-2} \leqq C_{0} \log M,
$$

and similarly,

$$
\left|\int_{\left\{X_{0} \backslash\{|x| \leqq|\alpha|\}\right\}} \Phi(x) d x\right| \leqq C \int_{\{|\alpha| \leqq|x| \leqq 2 M|\alpha|\}}|x|^{-2} d x \leqq C_{0} \log M .
$$

Equations (3.20), (3.22)-(3.25) imply

$$
\left.\left|\sum_{m} \Phi(|\alpha| m)\right| \alpha\right|^{2}-\left(6 / \pi^{2}\right) \int_{|x| \geqq|\alpha|} \Phi(x) d x \mid \leqq C\left(\left(N^{-1}+\varepsilon_{M N}\right)|\log | \alpha||+\log M\right),
$$

hence by (3.1),

$$
\left.|| \alpha\right|^{-1}\left(D_{e}(\alpha)-D_{e}(0)\right)-\left(6 / \pi^{2}\right) \int_{|x| \geqq|\alpha|} \Phi(x) d x \mid \leqq C\left(\left(N^{-1}+\varepsilon_{M N}\right)|\log | \alpha||+\log M\right) .
$$


For every $\delta>0$ we can choose $N$ such that $C N^{-1}<\delta$, and then $M>N$ such that $C \varepsilon_{M N}<\delta$, so that the RHS of (3.27) is less than $2 \delta|\log | \alpha||+C \log M$. Now we can choose $\varepsilon=1 / M^{N}$ so that $C \log M<\delta|\log | \alpha||$, when $|\alpha|<\varepsilon$. Hence by (3.27),

$$
\left.|| \alpha\right|^{-1}\left(D_{e}(\alpha)-D_{e}(0)\right)-\left(6 / \pi^{2}\right) \int_{|x| \geqq|\alpha|} \Phi(x) d x|<3 \delta| \log |\alpha| \mid,
$$

when $|\alpha|<\varepsilon$. This proves that

$$
|\alpha|^{-1}\left(D_{e}(\alpha)-D_{e}(0)\right)-\left(6 / \pi^{2}\right) \int_{|x| \geqq|\alpha|} \Phi(x) d x=o(|\log | \alpha||), \quad|\alpha| \rightarrow 0 .
$$

By (3.3),

$$
\begin{aligned}
\int_{|x| \geqq|\alpha|} \Phi(x) d x & =\int_{|x| \geqq|\alpha|}\left(U\left(2\left(x_{2} \zeta_{1}-x_{1} \zeta_{2}\right)\right)-U(0)\right)|x|^{-3} d x \\
& =\int_{|y| \geqq|\alpha|}\left(U\left(2 y_{1}\right)-U(0)\right)|y|^{-3} d y,
\end{aligned}
$$

where $y=\left(y_{1}, y_{2}\right)$ with

$$
\begin{aligned}
& y_{1}=x_{2} \zeta_{1}-x_{1} \zeta_{2}, \\
& y_{2}=x_{2} \zeta_{2}+x_{1} \zeta_{1}
\end{aligned}
$$

For small $\left|y_{1}\right|$,

$$
U\left(2 y_{1}\right)-U(0)=-2\left|y_{1}\right|+O\left(y_{1}^{2}\right) .
$$

A straightforward evaluation gives

hence

$$
\int_{1 \geqq|y| \geqq|\alpha|}\left|y_{1}\right||y|^{-3} d y=\int_{0}^{2 \pi} d \varphi \int_{|\alpha|}^{1} r d r r|\cos \varphi| r^{-3}=4|\log | \alpha||,
$$

$$
\int_{|y| \geqq|\alpha|}\left(U\left(2 y_{1}\right)-U(0)\right)|y|^{-3} d y=-8|\log | \alpha||+O(1),
$$

hence using the fact that $U(\cdot)$ is bounded,

$$
\int_{|x| \geqq|\alpha|} \Phi(x) d x=-8|\log | \alpha||+O(1) .
$$

Therefore from (3.28),

$$
|\alpha|^{-1}\left(D_{\mathrm{e}}(\alpha)-D_{\mathrm{e}}(0)\right)=-\left(48 / \pi^{2}\right)|\log | \alpha||+o(|\log | \alpha||),
$$

or in other words,

$$
\lim _{\alpha \rightarrow 0}(|\log | \alpha|| \cdot|\alpha|)^{-1}\left(D_{\mathrm{e}}(\alpha)-D_{\mathrm{e}}(0)\right)=-48 / \pi^{2} .
$$

The same considerations give

$$
\lim _{\alpha \rightarrow 0}(|\log | \alpha|| \cdot|\alpha|)^{-1}\left(D_{0}(\alpha)-D_{0}(0)\right)=-16 / \pi^{2} .
$$

The result for the odd part is three times less because the density of relatively prime odd pairs $k, l$ is $2 / \pi^{2}$, and not $6 / \pi^{2}$. From (3.30), (3.31),

$$
\lim _{\alpha \rightarrow 0}(|\log | \alpha|| \cdot|\alpha|)^{-1}(D(\alpha)-D(0))=-64 / \pi^{2} .
$$

For $\beta=0$ Theorem 1.1 is proved. 


\section{Proof of Theorem 1.1 for $\beta \neq 0$}

Let us partition all relatively prime pairs $k, l$ into subsets

$S_{r}=\left\{k, l\right.$ are relatively prime and $\left.l n_{1}-k n_{2} \equiv r \bmod 2 Q\right\}, \quad r=0,1, \ldots, 2 Q-1$.

We can rewrite $(2.17)$ as

with

$$
D_{\mathrm{e}}(\alpha)=\sum_{r} D_{e r}(\alpha)
$$

$$
D_{e r}(\alpha)=\sum_{S_{r}} U(w)\left(k^{2}+l^{2}\right)^{-3 / 2}
$$

Our aim is to estimate

$$
\begin{gathered}
D_{e r}(\alpha)-D_{e r}(\beta)=\sum_{S_{r}}(U(w)-U(v))\left(k^{2}+l^{2}\right)^{-3 / 2}, \\
w=2\left(l \alpha_{1}-k \alpha_{2}\right), \quad v=2\left(l \beta_{1}-k \beta_{2}\right)=Q^{-1}\left(l n_{1}-k n_{2}\right) \equiv Q^{-1} r \bmod 2 .
\end{gathered}
$$

Denote

$$
\delta=\alpha-\beta, \quad \zeta=|\delta|^{-1} \delta=\left(\zeta_{1}, \zeta_{2}\right), \quad \eta=\left(-\zeta_{2}, \zeta_{1}\right), \quad m=(k, l) \in \mathbf{Z}^{2} .
$$

Then (4.3) reduces to

where

$$
\begin{aligned}
|\delta|^{-1}\left(D_{e r}(\alpha)-D_{e r}(\beta)\right) & =\sum_{S_{r}}\left(U\left(Q^{-1} r+2|\delta| m \cdot \eta\right)-U\left(Q^{-1} r\right)\right)|m| \delta||^{-3}|\delta|^{2} \\
& =\sum_{S_{r}} \Phi_{r}(|\delta| m)|\delta|^{2}
\end{aligned}
$$

$$
\Phi_{r}(x)=\left(U\left(Q^{-1} r+2 x \cdot \eta\right)-U\left(Q^{-1} r\right)\right)|x|^{-3}, \quad x \cdot \eta=x_{1} \eta_{1}+x_{2} \eta_{2} .
$$

As in the proof of (3.28) we obtain now

$$
|\delta|^{-1}\left(D_{e r}(\alpha)-D_{e r}(\beta)\right)=d_{r} \int_{|x| \geqq|\delta|} \Phi_{r}(x) d x+o(|\log | \delta||),
$$

where $d_{r}$ is the density of $S_{r}$.

By Lemma $2.1 U(w) \in C^{\infty}([0,1])$, so when $r \neq 0, Q$,

Since

$$
\Phi_{r}(x)=C x \cdot \eta|x|^{-3}+O\left(|x|^{-1}\right), \quad|x| \rightarrow 0 .
$$

(4.5) implies

$$
\int_{1 \geqq|x| \geqq|\delta|} x \cdot \eta|x|^{-3} d x=0
$$

$$
|\delta|^{-1}\left(D_{e r}(\alpha)-D_{e r}(\beta)\right)=o(|\log | \delta||), \quad r \neq 0, Q .
$$

In the case when $r=0, Q, \Phi_{r}(x)=\Phi(x)$ and so by (3.29),

$$
\int_{|x| \geqq|\delta|} \Phi_{r}(x) d x=\int_{|x| \geqq|\delta|} \Phi(x) d x=-8|\log | \delta||+O(1),
$$

hence (4.5) implies

$$
|\delta|^{-1}\left(D_{e r}(\alpha)-D_{e r}(\beta)\right)=-8 d_{r}|\log | \delta||+o(|\log | \delta||), \quad r=0, Q .
$$

From (4.7), (4.8),

$$
|\delta|^{-1}\left(D_{\mathrm{e}}(\alpha)-D_{\mathrm{e}}(\beta)\right)=-8 \Delta|\log | \delta||+o(|\log | \delta||),
$$


where $\Delta=d_{0}+d_{Q}$ is the density of $k, l$ such that

and

$$
\operatorname{gcd}(k, l)=1
$$

$$
\ln _{1}-k n_{2} \equiv 0 \bmod Q \text {. }
$$

Similar considerations applying to $D_{\mathrm{o}}(\alpha)-D_{\mathrm{o}}(\beta)$ leads us to

$$
|\delta|^{-1}\left(D_{\mathrm{o}}(\alpha)-D_{\mathrm{o}}(\beta)\right)=-8\left(\Delta_{1}-\Delta_{2}\right)|\log | \delta||+o(|\log | \delta||),
$$

where $\Delta_{1,2}$ are the densities of odd relatively prime pairs $k, l$ such that

$$
\ln _{1}-k n_{2} \equiv 0, Q \bmod 2 Q,
$$

respectively.

From (4.9), (4.12) (remember $\delta=\alpha-\beta$ ),

$$
|\alpha-\beta|^{-1}(D(\alpha)-D(\beta))=-8\left(\Delta+\Delta_{1}-\Delta_{2}\right)|\log | \alpha-\beta||+o(|\log | \alpha-\beta||) .
$$

which implies

$$
\lim _{\alpha \rightarrow \beta}(|\alpha-\beta||\log | \alpha-\beta||)^{-1}(D(\alpha)-D(\beta))=-8\left(\Delta+\Delta_{1}-\Delta_{2}\right) .
$$

It remains to calculate $\Delta$ and the other densities in (4.15). $\Delta$ is the density of pairs $(k, l)$ satisfying (4.10) and (4.11). Since the highest common factor of $n_{1}$ and $n_{2}$ is prime to $Q$, the pairs $(k, l)$ satisfying (4.11) form an integer lattice of density $Q^{-1}$. Within this lattice, the condition (4.10) eliminates a fraction $f(p)$ of pairs, independently for every prime $p$. Therefore

with

$$
\Delta=\left(Q^{-1}\right) \prod_{p}(1-f(p))
$$

Since

$$
f(p)=p^{-1} \text { if } p \text { divides } Q, f(p)=p^{-2} \text { otherwise }
$$

$$
\prod_{p}\left(1-p^{-2}\right)=\left(6 / \pi^{2}\right)
$$

Eq. (4.16) with (1.5) gives

$$
\Delta=(Q r(Q))^{-1}\left(6 / \pi^{2}\right) .
$$

If we ignore the condition (4.13), the density of pairs of odd $(k, l)$ satisfying (4.11) and (4.10) is (4.16) with the factor $(1-f(2))$ arising from the prime $p=2$ replaced by $(1 / 4)$. Therefore

$$
\Delta_{1}+\Delta_{2}=(1 / 3) \Delta, \quad(Q \text { odd }) ; \Delta_{1}+\Delta_{2}=(1 / 2) \Delta,(Q \text { even }) .
$$

On the other hand, if $Q$ is odd, both $k$ and $l$ being odd, the condition (4.13) reduces to

$$
\left(n_{1}+n_{2}\right) \equiv(0,1)(\bmod 2)
$$

which implies

$$
\begin{array}{ll}
\Delta_{1}=(1 / 3) \Delta, \quad \Delta_{2}=0 \quad & \left(\left(n_{1}+n_{2}\right) \text { even }\right) \\
\Delta_{1}=0, \quad \Delta_{2}=(1 / 3) \Delta \quad\left(\left(n_{1}+n_{2}\right) \text { odd }\right)
\end{array}
$$


Finally, if $Q$ is even, at least one of $n_{1}$ and $n_{2}$ must be odd, and the two cases (4.13) will be satisfied equally often, so that

$$
\Delta_{1}=\Delta_{2}=(1 / 4) \Delta, \quad(Q \text { even }) .
$$

Putting together (4.20), (4.22) and (4.23), we obtain

$$
\Delta+\Delta_{1}-\Delta_{2}=(C / 3) \Delta
$$

with $C$ given by (1.6). Putting together (4.24) with (4.15) and (4.19), we obtain (1.2) with (1.4). Theorem 1.1 is proved.

\section{Appendix. Proof of Lemma 2.1}

We have

with

$$
\begin{aligned}
& U(w)=\frac{1}{2 \pi^{5 / 2}} \int_{0}^{\infty} a^{-5 / 2}\left(F_{a}(w) F_{a}(0)-1\right) d a \\
& V(w)=\frac{1}{2 \pi^{5 / 2}} \int_{0}^{\infty} a^{-5 / 2} G_{a}(w) G_{a}(0) d a
\end{aligned}
$$

$$
\sum_{x} \exp \left(-x^{2} / a\right) e(x w)=F_{a}(w) \text { or } G_{a}(w),
$$

where the sum is over integer $x$ for $F_{a}$ and over half-odd-integer $x$ for $G_{a}$. By the Poisson summation formula, (A.1) gives

$$
\begin{aligned}
& F_{a}(w)=(\pi a)^{1 / 2} \sum_{p=-\infty}^{\infty} \exp \left(-\pi^{2} a(p+w)^{2}\right), \\
& G_{a}(w)=(\pi a)^{1 / 2} \sum_{p=-\infty}^{\infty}(-1)^{p} \exp \left(-\pi^{2} a(p+w)^{2}\right) .
\end{aligned}
$$

Divide integrals into $a<1$ and $a>1$. Integrals $a<1$ are analytic in $w$ by (A.1). Integrals $a>1$ are analytic in $w$ by (A.2) when $w$ is real and not integer. So $U$ and $V$ are analytic on $(0,1)$. If $w$ is close to 0 , we have by (A.2),

$$
F_{a}(w) F_{a}(0), G_{a}(w) G_{a}(0)=\pi a \exp \left(-\pi^{2} a w^{2}\right)+R_{a}(w),
$$

where $\int_{1}^{\infty} a^{-5 / 2} R_{a}(w) d a$ is even and analytic in $w$. Only the first term contributes to $U^{\prime}(w), V^{\prime}(w)$ as $w \rightarrow 0$, and gives

$$
\begin{gathered}
U^{\prime}(w), V^{\prime}(w) \sim-\pi^{1 / 2} w \int_{1}^{\infty} a^{-1 / 2} \exp \left(-\pi^{2} a w^{2}\right) d a \\
=-2 \pi^{-1 / 2} \frac{w}{|w|} \int_{\pi w}^{\infty} \exp \left(-b^{2}\right) d b
\end{gathered}
$$

which is analytic at $w=+0$ with $U^{\prime}(+0)=V^{\prime}(+0)=-1$.

Acknowledgement. The authors thank E. Bombieri and J. Lebowitz for useful discussions of the paper. This work was partly supported by a grant from The Ambrose Monell Foundation. 


\section{References}

[B] Bleher, P.M.: On the distribution of the number of lattice points inside a family of convex ovals. Duke Math. J. 67, 461-481 (1992)

[BCDL] Bleher, P.M., Cheng, Zh., Dyson, F.J., Lebowitz, J.L.: Distribution of the error term for the number of lattice points inside a shifted circle. Commun. Math. Phys. 154, 433-469 (1993)

[BD] Bleher, P.M., Dyson, F.J.: Mean square value of exponential sums related to representation of integers as sum of two squares. Preprint IASSNS-HEP-92/84, Institute for Advanced Study, Princeton, 1992

[C] Cramér, H.: Über zwei Sätze von Herrn G.H. Hardy. Math. Zeit. 15, 201-210 (1922)

[H-B] Heath-Brown, D.R.: The distribution and moments of the error term in the Dirichlet divisor problem. Acta Arithmetica 60, 389-415 (1992)

[K] Kendall, D.G.: On the number of lattice points inside a random oval. Quart. J. Math. (Oxford) 19, 1-26 (1948)

Communicated by T. Spencer 
